Johnny Alexander Bastidas Otero

\title{
Algoritmos genéticos aplicados à solução do problema inverso biomagnético
}

Dissertação de Mestrado

Dissertação apresentada como requisito parcial para obtenção do grau de Mestre pelo Programa de PósGraduação em Engenharia Elétrica do Departamento de Engenharia Elétrica da PUC-Rio.

Orientador: Prof. Eduardo Costa da Silva Co-Orientadora: Prof ${ }^{a}$. Elisabeth Costa Monteiro 
Johnny Alexander Bastidas Otero

\section{Algoritmos genéticos aplicados à solução do problema inverso biomagnético}

Dissertação apresentada como requisito parcial para obtenção do grau de Mestre pelo Programa de Pós-Graduação em Engenharia Elétrica do Departamento de Engenharia Elétrica do Centro Técnico Científico da PUC-Rio. Aprovada pela Comissão Examinadora abaixo assinada.

Prof. Eduardo Costa da Silva

Orientador

Departamento de Engenharia Elétrica - PUC-Rio

Profa. Elisabeth Costa Monteiro

Co-orientadora

Programa de Pós-Graduação em Metrologia - PUC-Rio

Prof. Carlos Roberto Hall Barbosa Programa de Pós-Graduação em Metrologia - PUC-Rio

Prof $^{a}$. Karla Tereza Figueiredo Leite

UERJ

Prof. José Franco Machado do Amaral

UERJ

Prof. Márcio da Silveira Carvalho

Coordenador Setorial do Centro Técnico Científico - PUC-Rio

Rio de Janeiro, 22 de setembro de 2016 
Todos os direitos reservados. É proibida a reprodução total ou parcial do trabalho sem autorização da universidade, do autor e do orientador.

Johnny Alexander Bastidas Otero

Graduou-se em Engenharia Eletrônica pela Universidade de Nariño - Colômbia 2012.

Ficha Catalográfica

Bastidas Otero, Johnny Alexander

Algoritmos genéticos aplicados à solução do problema inverso biomagnético / Johnny Alexander Bastidas Otero; orientador: Eduardo Costa da Silva; co-orientadora: Elisabeth Costa Monteiro. - 2016.

$157 \mathrm{f}$. : il. color. ; $30 \mathrm{~cm}$

Dissertação (mestrado)-Pontifícia Universidade Católica do Rio de Janeiro, Departamento de Engenharia Elétrica, 2016.

Inclui bibliografia

1. Engenharia elétrica - Teses. 2. Problema inverso biomagnético. 3. Biomagnetismo. 4. Algoritmos genéticos. 5. Dipolos elétricos. 6. Magnetocardiografia I. Bastidas Otero, Johnny Alexander. II. Monteiro, Elisabeth Costa. III. Pontifícia Universidade Católica do Rio de Janeiro. Departamento de Engenharia Elétrica. IV. Título.

CDD: 621.3 
A Deus, que me concedeu um caminho e ilumina cada passo do meu caminhar, a minha mãe, modelo de devoção, compreensão e dedicação, a meu pai, exemplo de superação, disciplina e segurança, a minha irmã pela ajuda incondicional e a toda minha família pelo apoio. 


\section{Agradecimentos}

Ao meu orientador professor Dr. Eduardo Costa da Silva, pela aceitação, acreditação, auxilio, ensinamentos, dedicação, paciência, aportes, revisões, sugestões e contribuições substanciais ao trabalho, obrigado pela amizade e apoio incondicional. É uma pessoa admirável que respeito e aprecio e não tenho mais que agradecimentos e estima.

À minha co-orientadora Professora Dra. Elisabeth Costa Monteiro e meu professor Dr. Carlos Roberto Hal Barbosa, pela ajuda incondicional, dedicação, aportes, revisão, auxilio, assessoramento, gentileza e apoio. Suas contribuições foram fundamentais no desenvolvimento deste trabalho.

Aos membros da Comissão Examinadora por suas contribuições a este trabalho.

Aos Professores da PUC-Rio, dos Departamentos de Engenharia Elétrica e Engenharia de Controle e Automação, e Metrologia, pela sólida formação acadêmica.

À PUC-Rio, por acreditar na pesquisa científica como instrumento de desenvolvimento social e evolução humana.

À CAPES, pelos auxílios financeiros concedidos.

Aos meus amigos, pelo apoio, ajuda, tolerância, compreensão e por todos os momentos compartidos. Foram, são e serão parte de minha vida e da minha família até que Deus permita. 


\section{Resumo}

Bastidas Otero, Johnny Alexander; Costa da Silva, Eduardo (Orientador); Costa Monteiro; Elizabeth (Co-orientadora). Algoritmos genéticos aplicados à solução do problema inverso biomagnético. Rio de Janeiro, 2016. 157p. Dissertação de Mestrado - Departamento de Engenharia Elétrica, Pontifícia Universidade Católica do Rio de Janeiro.

Sinais bioelétricos fornecem informações importantes sobre a função fisiológica de muitos organismos vivos. Em magnetismo, denomina-se problema direto aquele em que se determina o campo magnético a partir do conhecimento da fonte de corrente que o gerou. Por outro lado, existem situações em que se deseja determinar a fonte de corrente a partir de valores de campo magnético medidos. Esse tipo de problema é usual em Biomagnetismo e é denominado problema inverso. Por exemplo, com base em medições do campo magnético cardíaco é possível inferir sobre a atividade elétrica, no tecido cardíaco, que foi responsável por sua geração. Este trabalho propõe, apresenta e discute uma nova técnica destinada a resolver o problema biomagnético inverso, por meio de algoritmos genéticos. Objetiva-se estimar a posição, a orientação e a magnitude dos dipolos de corrente equivalentes, responsáveis pela geração de mapas de campos biomagnéticos obtidos experimentalmente por meio de medições realizadas em corações isolados de coelho utilizando um sistema SQUID de 16 canais. O algoritmo busca identificar a distribuição de dipolos que melhor se ajusta aos dados experimentais, objetivando minimizar o erro entre o mapa de campo magnético medido e o obtido por meio das soluções estimadas. O conhecimento dos parâmetros dos dipolos de corrente, em diferentes instantes de tempo, permite a correta interpretação e análise da informação médica obtida a partir dos campos biomagnéticos medidos experimentalmente, auxiliando na definição de diagnósticos e orientação de abordagens terapêuticas.

\section{Palavras-chave}

Problema inverso biomagnético; Biomagnetismo; Algoritmos genéticos; Dipolos elétricos; Magnetocardiografia. 


\section{Abstract}

Bastidas Otero, Johnny Alexander; Costa da Silva, Eduardo (Advisor); Costa Monteiro; Elizabeth (Co-Advisor). Genetic algorithms applied to the solution of the biomagnetic inverse problem. Rio de Janeiro, 2016. 157p. MSc. Dissertation - Departamento de Engenharia Elétrica, Pontifícia Universidade Católica do Rio de Janeiro.

Bioelectric signals provide important information about the physiological function of many living organisms. In magnetism, the so-called direct problem deals with the determination of the magnetic field associated to well-known current sources. On the other hand, there are situations where it is necessary to determine the current source responsible for the generation of a measured magnetic field. This type of problem is common in Biomagnetism and is called inverse problem. For example, based on cardiac magnetic field measurements it is possible to infer the electrical activity in the heart tissue, responsible for its generation. This work proposes, presents and discusses a new technique designed to solve the biomagnetic inverse problem by genetic algorithms. It is intended to estimate the position, orientation and magnitude of the equivalent current dipoles, responsible for the generation of biomagnetic field maps measured with a 16 channel SQUID system. The algorithm attempts to identify the distribution of dipoles that best fits the measured experimental data, aiming at minimizing the error between the experimental magnetic field maps and those obtained by the estimated solutions. The experimental data analyzed in this study were acquired by measurements in isolated rabbit hearts. The knowledge of parameters of current dipoles at different instants of time allows the correct interpretation and analysis of medical information obtained from the experimentally measured biomagnetic fields, providing diagnosis and guiding therapeutic procedures.

\section{Keywords}

Biomagnetic inverse problem; Biomagnetism; Genetic Algorithm; Electric dipole; Magnetocardiography. 


\section{Sumário}

1 Introdução 20

1.1. Contextualização e Motivação 20

1.2. Objetivos 22

1.3. Organização da Dissertação 23

2 Biomagnetismo 25

2.1. Sistemas de medição Biomagnética 28

2.2. Interferência Magnética e Ruído Ambiental 35

2.2.1. Blindagem Magnética 35

2.2.2. Gradiômetros $\quad 37$

¿ 2.3. Sistemas Comerciais para Medições Biomagnéticas 39

2.3.1. Twin-Dewar $\quad 39$

2.3.2. babySQUID 40

2.3.3. ARGOS 200

2.3.4. Micro-SQUIDs 42

2.4. Problema Inverso 44

2.5. Cardiomagnetismo 50

3 Algoritmos Genéticos 53

3.1. Representação ou Codificação 55

3.2. Avaliação 56

3.3. Seleção 57

3.4. Operadores Genéticos $\quad 59$

3.5. Parâmetros da Evolução 62

4 Metodologia $\quad 65$

4.1. Modelagem Matemática 65

4.2. Dados Experimentais 68

4.3. Problema Direto 74

4.4. Algoritmo Genético 79 
5 Resultados Experimentais e Discussão 84

5.1. Dipolos livres 84

5.1.1. População com tamanho fixo 85

5.1.1.1. Um Dipolo 85

5.1.1.2. Múltiplos dipolos 93

5.1.2. População com tamanho variável 97

5.1.3. Crossover Heurístico adaptativo 101

5.2. Dipolos semi-livres 107

5.3. Dipolos fixos 110

5.4. Comparação das Técnicas 112

5.5. Avaliação da generalidade da técnica desenvolvida 114

5.6. Estudos Simulados para Avaliação do Método Desenvolvido 116

6 Conclusões e Trabalhos Futuros 141

$\begin{array}{ll}\text { Referências bibliográficas } & 145\end{array}$ 


\section{Lista de Figuras}

Figura 1 - Densidades de fluxo biomagnético em comparação com densidades de fluxo de fontes de interferência magnética.

Figura 2 - Campos biomagnéticos e suas respectivas faixas de frequência [12]. 27

Figura 3 - Diferentes tipos de Magnetômetros e uma comparação entre suas faixas de detecção.

Figura 4 - Diagrama de blocos de um SQUID típico.

Figura 5 - Sistema multicanal de Magnetocardiografia com 99 canais [1].

Figura 6 - Câmara blindada BMSR-2, (a) fase de construção,

destacando-se as camadas de $\mu$-metal, (b) vista exterior da estrutura pronta para operação [75].

Figura 7 - Gradiômetros SQUID: (a) ordem zero, (b) $1^{\mathrm{a}}$ ordem, (c) $2^{\mathrm{a}}$ ordem. 38

Figura 8 - Biomagnetômetro Twin-Dewar de 62 canais [1].

Figura 9 - Biomagnetômetro Neonatal BabySQUID [1].

Figura 10 - Magnetocardiógrafo ARGOS 200 [1].

Figura 11 - Micro-SQUID Jena: a) arranjo dos gradiômetros;

b) apresentação de uma medida de MEG típica, por meio do emprego do software CURRY [1].

Figura 12 - Comparação entre o problema inverso e o problema direto.

Figura 13 - Fluxograma típico de um algoritmo evolucionário.

Figura 14 - Representação da seleção por roleta para 5 indivíduos.

Figura 15 - Representação do cruzamento de um ponto.

60

Figura 16 - Representação do cruzamento de dois pontos.

Figura 17 - Representação do cruzamento uniforme.

Figura 18 - Representação da operação genética de mutação.

Figura 19 - Representação simplificada de um sistema unidimensional para calcular o campo magnético gerado por um dipolo de corrente.

Figura 20 - Amostra de tecido atrial isolado de coelho mantida em uma configuração plana na superfície de um recipiente não-magnético para a realização das medições biomagnéticas, utilizando o sistema SQUID de 16 canais do Biomagnetisches Zentrum. 
Figura 21 - Resultados experimentais referentes a PB1: (a) Mapas de isocampo magnético em instantes de tempo consecutivos, e

(b) magnitude dos dipolos correspondentes a cada mapa apresentado.

Figura 22 - Resultados experimentais referentes a PB2: (a) Mapas de isocampo magnético em instantes de tempo consecutivos, e

(b) magnitude dos dipolos em função do tempo.

Figura 23 - Resultados experimentais referentes a PB3: (a) Mapas de isocampo magnético em instantes de tempo consecutivos, e

(b) magnitude dos dipolos em função do tempo.

Figura 24 - Mapa de campo magnético de um dipolo, com: $\rho=10 \mu \mathrm{A} \cdot \mathrm{m}$, $\mathrm{X}=0, \mathrm{Y}=0, \alpha=45^{\circ}$.

Figura 25 - Mapa de campo magnético de um dipolo, com: $\rho=5 \mu \mathrm{A} \cdot \mathrm{m}$, $\mathrm{X}=32 \mathrm{~mm}, \mathrm{Y}=32 \mathrm{~mm}, \alpha=225^{\circ}$.

Figura 26 - Mapa de campo magnético de um dipolo, com: $\rho=1 \mu \mathrm{A} \cdot \mathrm{m}$, $\mathrm{X}=12,5 \mathrm{~mm}, \mathrm{Y}=12,5 \mathrm{~mm}, \alpha=180^{\circ}$.

Figura 27 - Mapa de campo magnético de um dipolo, com: $\rho=25 \mu \mathrm{A} \cdot \mathrm{m}$, $\mathrm{X}=13 \mathrm{~mm}, \mathrm{Y}=18 \mathrm{~mm}, \alpha=315^{\circ}$.

Figura 28 - Mapas de campo magnético de: (a) 2 dipolos, (b) 4 dipolos,

(c) 9 dipolos, (d) 16 dipolos; com os parâmetros da Tabela 1.

Figura 29 - Representação gráfica das três propostas de configuração

do espaço de soluções do problema analisado: (a) dipolos livres,

(b) dipolos semi-livres, (c) dipolos fixos.

Figura 30 - Média do erro em cada um dos instantes analisados, para um dipolo livre.

Figura 31 - Desvio padrão em cada um dos instantes analisados,

para um dipolo livre.

Figura 32 - Erro absoluto ótimo em cada instante de tempo analisado, para um dipolo livre.

Figura 33 - Comparação entre os mapas normalizados de campo magnético, em 200ms: (a) mapa experimental $B_{Z n o r m}$ e (b) mapa computacionalmente estimado $B_{Z A G n o r m}$; para um único dipolo livre. 
Figura 34 - Comparação entre os mapas normalizados de campo magnético, em 100ms: (a) mapa experimental $B_{Z n o r m}$ e (b) mapa computacionalmente estimado $B_{Z A G n o r m}$; para um único dipolo livre.

Figura 35 - Representação dos dipolos equivalentes estimado pelo AG, para os instantes de tempo analisados nas Figura 33 e Figura 34:

(a) $200 \mathrm{~ms}$ e (b) $100 \mathrm{~ms}$.

Figura 36 - Representação dos dipolos equivalentes gerados pelo AG:

(a) comprimento mínimo da seta e (b) comprimento máximo da seta.

Figura 37 - Comparação entre os parâmetros do dipolo equivalente estimado por a técnica implementada em [90] (esquerda) e a técnica desenvolvida nesta dissertação (direita), nos instantes de tempo: (a) $132 \mathrm{~ms}$,

(b) $152 \mathrm{~ms}$, (c) $200 \mathrm{~ms}$, (d) $224 \mathrm{~ms}$.

Figura 38 - Representação dos dipolos estimados pelo AG em cada um dos 43 instantes de tempo, entre $100 \mathrm{~ms}$ e $268 \mathrm{~ms}$.

Figura 39 - Média dos erros, em valores percentuais, e tempo médio total de processamento, em horas, em função da quantidade de dipolos $\left(\mathrm{n}^{2}\right)$. Figura 40 - Comparação entre os erros absolutos ótimos alcançados pelos AG baseados em 1 e 4 dipolos livres, em cada instante de tempo analisado. Figura 41 - Comparação dos mapas magnéticos obtidos para o instante de tempo de 112 ms: (a) mapa experimental, (b) AG com 1 dipolo livre, e (c) AG com 4 dipolos livres.

Figura 42 - Comparação dos mapas magnéticos obtidos para o instante de tempo 164 ms: (a) mapa experimental, (b) AG com 1 dipolo livre, e (c) AG com 4 dipolos livres.

Figura 43 - Representação dos dipolos gerados pelo AG para o caso da Figura 41 com (a) 1 dipolo livre, e (b) 4 dipolos livres.

Figura 44 - Representação dos dipolos gerados pelo AG para o caso da Figura 42 com (a) 1 dipolo livre, e (b) 4 dipolos livres.

Figura 45 - Média dos erros absolutos, em valores percentuais, em função da quantidade de dipolos $\left(n^{2}\right)$; para tamanhos de população iguais a

(a) 100 indivíduos, (b) 6 vezes a quantidade de variáveis e

(c) 10 vezes a quantidade de variáveis. 
Figura 46 - Tempo médio total de processamento, em horas, em função da quantidade de dipolos: esquerda 1-16, direita 25-64; para tamanhos de população iguais a (a) 100 indivíduos, (b) 6 vezes a quantidade de variáveis e (c) 10 vezes a quantidade de variáveis.

Figura 47 - Fluxograma da técnica de crossover heurístico adaptativo.

Figura 48 - Comparação dos erros médios obtidos por meio da adoção da abordagem de AG com $R$ fixo $(R=2)$ e baseados no crossover heurístico adaptativo de $R=2-6-2$, em função da quantidade de dipolos. Figura 49 - Comparação dos tempos totais de processamento dos AGs implementados com $R=2$ e $R=2-6-2$, em função da quantidade de dipolos: (a) 1-16 (b) 25-64.

Figura 51 - Comparação entre um mapa de campo magnético experimental (a) e o mapa obtido pelos dipolos calculados pelo AG, empregando-se 16 dipolos livres (b).

Figura 52 - Comparação entre um mapa de campo magnético experimental (a) e o mapa obtido pelos dipolos calculados pelo AG, empregando-se 25 dipolos livres (b).

Figura 53 - Comparação entre um mapa de campo magnético experimental (a) e o mapa obtido pelos dipolos calculados pelo AG, empregando-se 36 dipolos livres (b).

Figura 54 - Comparação entre um mapa de campo magnético experimental (a) e o mapa obtido pelos dipolos calculados pelo AG, empregando-se 49 dipolos livres (b).

Figura 55 - Comparação entre um mapa de campo magnético experimental (a) e o mapa obtido pelos dipolos calculados pelo AG, empregando-se 64 dipolos livres (b).

Figura 56 - Comparação entre o erro médio obtido pelo algoritmo de busca aleatória e aquele alcançado pelo AG, para diferentes quantidades de dipolos livres.

Figura 57 - Comparação entre um mapa de campo magnético experimental (a) e o mapa obtido pelos dipolos calculados pelo AG, empregando-se 9 dipolos semi-livres (b). 
Figura 58 - Comparação entre o erro médio obtido pelo algoritmo de busca aleatória e aquele alcançado pelo AG, para diferentes quantidades de dipolos semi-livres.

Figura 59 - Comparação dos tempos totais de processamento dos AGs e Busca Aleatória, em função da quantidade de dipolos semi-livres:

(a) $1-16$ (b) $25-64$.

Figura 60 - Comparação entre um mapa de campo magnético

experimental (a) e o mapa obtido pelos dipolos calculados pelo AG, empregando-se 9 dipolos fixos (b).

Figura 61 - Comparação entre o erro médio obtido pelo algoritmo de busca aleatória e aquele alcançado pelo AG, para diferentes quantidades de dipolos fixos.

Figura 62 - Comparação dos tempos totais de processamento dos AGs e Busca Aleatória, para dipolos fixos em função da quantidade de dipolos:

(a) 1-16 (b) 25-64.

Figura 63 - Comparação do erro obtido pelas três configurações de AG avaliadas, dipolos livres, semi-livres e fixos.

Figura 64 - Comparação do tempo total de processamento associado a cada uma das três configurações de AG avaliadas, dipolos livres, semi-livres e fixos: (a)1-16, (b) 25-64.

Figura 65 - Médias dos erros em função da quantidade de dipolos livres adotada, para cada um dos 3 casos analisados.

Figura 66 - Tempo médio de processamento por cada mapa para cada um dos 3 casos analisados, em função da quantidade de dipolos livres adotada: (a) 1 a 16 e (b) 25 a 64.

Figura 67 - Exemplo 1: Mapas de campo associados a um único dipolo de corrente: (a) mapa de referência (problema direto), (b) mapa gerado pelo dipolo elétrico retornado pelo AG (problema inverso).

Figura 68 - Exemplo 2: Mapas de campo associados a um único dipolo de corrente: (a) mapa de referência (problema direto), (b) mapa gerado pelo dipolo elétrico retornado pelo AG (problema inverso). 
Figura 69 - Exemplo 3: Mapas de campo associados a um único dipolo de corrente: (a) mapa de referência (problema direto), (b) mapa gerado pelo dipolo elétrico retornado pelo AG (problema inverso).

Figura 70 - Exemplo 1: Mapas de campo associados a dois dipolos de corrente: (a) mapa de referência (problema direto), (b) mapa gerado pelos dipolos elétricos retornados pelo AG (problema inverso).

Figura 71 - Exemplo 2: Mapas de campo associados a dois dipolos de corrente: (a) mapa de referência (problema direto), (b) mapa gerado pelos dipolos elétricos retornados pelo AG (problema inverso). Figura 72 - Exemplo 3: Mapas de campo associados a dois dipolos de corrente: (a) mapa de referência (problema direto), (b) mapa gerado pelos dipolos elétricos retornados pelo AG (problema inverso). Figura 73 - Exemplo 4: Mapas de campo associados a dois dipolos de corrente: (a) mapa de referência (problema direto), (b) mapa gerado pelos dipolos elétricos retornados pelo AG (problema inverso). Figura 74 - Exemplo 5: Mapas de campo associados a dois dipolos de corrente: (a) mapa de referência (problema direto), (b) mapa gerado pelos dipolos elétricos retornados pelo AG (problema inverso). Figura 75 - Exemplo 6: Mapas de campo associados a dois dipolos de corrente: (a) mapa de referência (problema direto), (b) mapa gerado pelos dipolos elétricos retornados pelo AG (problema inverso). Figura 76 - Exemplo 1: Mapas de campo associados a quatro dipolos de corrente dispostos na configuração 1: (a) mapa de referência (problema direto), (b) mapa gerado pelos dipolos elétricos retornados pelo $\mathrm{AG}$ (problema inverso).

Figura 77 - Exemplo 2: Mapas de campo associados a quatro dipolos de corrente dispostos na configuração 1: (a) o mesmo mapa de referência analisado na Figura 76 (problema direto), (b) mapa gerado pelos dipolos elétricos retornados pelo AG (problema inverso). 
Figura 78 - Exemplo 3: Mapas de campo associados a quatro dipolos de corrente dispostos na configuração 2: (a) mapa de referência (problema direto), (b) mapa gerado pelos dipolos elétricos retornados pelo AG (problema inverso).

Figura 79 - Exemplo 4: Mapas de campo associados a quatro dipolos de corrente dispostos na configuração 2: (a) o mesmo mapa de referência analisado na Figura 78 (problema direto), (b) mapa gerado pelos dipolos elétricos retornados pelo AG (problema inverso).

Figura 80 - Exemplo 5: Mapas de campo associados a quatro dipolos de corrente dispostos na configuração 3: (a) mapa de referência (problema direto), (b) mapa gerado pelos dipolos elétricos retornados pelo AG (problema inverso).

Figura 81 - Exemplo 6: Mapas de campo associados a quatro dipolos de corrente dispostos na configuração 3: (a) o mesmo mapa de referência analisado na Figura 80 (problema direto), (b) mapa gerado pelos dipolos elétricos retornados pelo AG (problema inverso). Figura 82 - Representação esquemática do método proposto para reduzir a dispersão das soluções obtidas para o problema inverso.

Figura 83 - Exemplo 1: Mapas de campo associados a quatro dipolos de corrente, $E_{t}=0.9766 \%$ : (a) o mesmo mapa de referência analisado nas Figuras 76 e 77 (problema direto), (b) mapa gerado pelos dipolos elétricos retornados pela configuração alternativa do AG (problema inverso). Figura 84 - Exemplo 2: Mapas de campo associados a quatro dipolos de corrente, $E_{t}=0.12735 \%$ : (a) o mesmo mapa de referência analisado nas Figuras 78 e 79 (problema direto), (b) mapa gerado pelos dipolos elétricos retornados pela configuração alternativa do AG (problema inverso).

Figura 85 - Exemplo 3: Mapas de campo associados a quatro dipolos de corrente, $E_{t}=0.56419 \%$ : (a) o mesmo mapa de referência analisado nas Figuras 80 e 81 (problema direto), (b) mapa gerado pelos dipolos elétricos retornados pela configuração alternativa do AG (problema inverso). 
Figura 86 - Mapas de campo associados ao caso exemplificado na Figura 83, para cada uma das alturas analisadas, na coluna (a) são apresentados os mapas de referência (problema direto) e na coluna (b) são apresentados os mapas correspondentes gerados pelos dipolos elétricos retornados pelo AG (problema inverso).

Figura 87 - Mapas de campo associados ao caso exemplificado na Figura 84, para cada uma das alturas analisadas, na coluna (a) são apresentados os mapas de referência (problema direto) e na coluna (b) são apresentados os mapas correspondentes gerados pelos dipolos elétricos retornados pelo AG (problema inverso). Figura 88 - Mapas de campo associados ao caso exemplificado na Figura 85, para cada uma das alturas analisadas, na coluna (a) são apresentados os mapas de referência (problema direto) e na coluna (b) são apresentados os mapas correspondentes gerados pelos dipolos elétricos retornados pelo AG (problema inverso). Figura 89 - Exemplo 1: Mapas de campo associados a quatro dipolos de corrente, $E_{t}=6.1523 \%$ : (a) o mesmo mapa de referência analisado nas Figuras 76 e 77 (problema direto), (b) mapa gerado pelos dipolos elétricos retornados pela configuração alternativa do AG (problema inverso). 133 Figura 90 - Exemplo 2: Mapas de campo associados a quatro dipolos de corrente, $E_{t}=6.9876 \%$ : (a) o mesmo mapa de referência analisado nas Figuras 78 e 79 (problema direto), (b) mapa gerado pelos dipolos elétricos retornados pela configuração alternativa do AG (problema inverso). 134 Figura 91 - Exemplo 3: Mapas de campo associados a quatro dipolos de corrente, $E_{t}=0.62154 \%$ : (a) o mesmo mapa de referência analisado nas Figuras 80 e 81 (problema direto), (b) mapa gerado pelos dipolos elétricos retornados pela configuração alternativa do AG (problema inverso). 134 Figura 92 - Mapas de campo associados ao caso exemplificado na Figura 89, para cada uma das alturas analisadas, na coluna (a) são apresentados os mapas de referência (problema direto) e na coluna (b) são apresentados os mapas correspondentes gerados pelos dipolos elétricos retornados pelo AG (problema inverso). 
Figura 93 - Mapas de campo associados ao caso exemplificado na Figura 90, para cada uma das alturas analisadas, na coluna (a) são apresentados os mapas de referência (problema direto) e na coluna (b) são apresentados os mapas correspondentes gerados pelos dipolos elétricos retornados pelo AG (problema inverso).

Figura 94 - Mapas de campo associados ao caso exemplificado na Figura 91, para cada uma das alturas analisadas, na coluna (a) são apresentados os mapas de referência (problema direto) e na coluna (b) são apresentados os mapas correspondentes gerados pelos dipolos elétricos retornados pelo AG (problema inverso).

Figura 95 - Mapas de campo associados aos casos previamente exemplificados nas Figura 89 ( $d=1$ a $5 \mathrm{~mm}$ ), agora considerando-se $d=1 \mathrm{~mm}$ : (a) mapa de referência (problema direto) e (b) mapa correspondente gerado pelos dipolos elétricos retornados pelo AG (problema inverso).

Figura 96 - Mapas de campo associados aos casos previamente exemplificados nas Figura 90 ( $d=1$ a $5 \mathrm{~mm}$ ), agora considerando-se $d=1 \mathrm{~mm}$ : (a) mapa de referência (problema direto) e (b) mapa correspondente gerado pelos dipolos elétricos retornados pelo AG (problema inverso).

Figura 97 - Mapas de campo associados aos casos previamente exemplificados nas Figura 91 ( $d=1$ a $5 \mathrm{~mm}$ ), agora considerando-se $d=1 \mathrm{~mm}$ : (a) mapa de referência (problema direto) e (b) mapa correspondente gerado pelos dipolos elétricos retornados pelo AG (problema inverso). 


\section{Lista de Tabelas}

Tabela 1 - Parâmetros de múltiplos dipolos representados na Figura 28.

Tabela 2 - Comparação entre os valores de referência e aqueles obtidos

pelo AG, para a situação de mapas de campo gerados por um dipolo único.

Tabela 3 - Comparação entre os valores dos parâmetros de referência e aqueles obtidos pelo AG, para a situação de mapas de campo gerados por dois dipolos.

Tabela 4 - Configurações dos parâmetros dos 4 dipolos utilizados para criar os mapas de referência. 